\title{
PULSE COUNTING IN ANODIC BREAKDOWN MEASUREMENTS
}

\author{
J. M. ALBELLA, I. MONTERO, J. BAONZA and J. M. MARTÍNEZ-DUART \\ Departamento de Física Aplicada and Instituto de Física del Estado Sólido (C.S.I.C.) Universidad Autónoma de \\ Madrid. Cantoblanco (Madrid) Spain
}

(Received August 1, 1979; in final form April 23, 1980)

\begin{abstract}
An electronic method, based on the differentiation of the potential curve during anodization at a constant current, is presented for evaluating the number of breakdown events in the scintillation of anodic oxides. This simple method facilitates the study of the breakdown phenomenon in anodic oxides as a function of the anodization current density, nature and resistivity of the electrolyte, etc. The results are briefly discussed according to a proposed model of the scintillation mechanism.
\end{abstract}

\section{INTRODUCTION}

Measurements of the breakdown or scintillation potential during the anodic oxidation of valve metals (e.g. $\mathrm{Al}, \mathrm{Ta}, \mathrm{Ti}$ ) are of great interest both from a basic point of view and in industrial applications, such as in the fabrication of electrolytic capacitors. During the last few years a considerable effort has been made in the development of electrolytic capacitors with a high nominal voltage. However, it is not yet clear how the breakdown voltage depends on certain anodization parameters such as resistivity and nature of the electrolyte, current density, etc. ${ }^{1,2}$ This is in part due to the lack of results on the breakdown process itself, i.e., after the first sparkvoltage has been reached.

When the scintillation potential is reached during the anodic oxidation, the voltage across the oxide levels off and starts to show small oscillations or pulses which may be attributed to small and localized breakdown phenomena. ${ }^{3,4}$ Consequently, the oxide is damaged. This damage is in the form of pores and fissures, sometimes distributed into branches, which can be studied by scanning electron microscopy. ${ }^{5}$

In this paper we present a method which facilitates a count of the pulses produced by the breakdown events during anodization to voltages beyond the first-scintillation voltage. This simple method, which does not involve any new electronic technique, can be applied to different types of samples such as regular stock tantalum foil, pressed tantalum pellets and high capacitance etched aluminum foil.

\section{EXPERIMENTAL METHCD}

The method is based upon the differentiation of the potential curve $V=V(t)$, in the anodization at a constant current, by means of an RC circuit. This allows the separation in the $V=V(t)$ curve of the fairly small breakdown peaks from the much slower and linear variation of the potential due to an increase in the thickness of the oxide. The peaks in the $d V(t) / d t$ curve are subsequently enlarged by an operational amplifier so that they can be conveniently processed and counted.

A block diagram of the electronic device, together with the modifications undergone by the input signal after each stage, is outlined in Figure 1. Once the signal has gone through the differentiating RC circuit, it is subsequently amplified by a variable gain operational amplifier. The resulting signal excites a monostable oscillator giving rise to a rectangular pulse. Finally, the output of the oscillator is taken to a conventional pulse counter.

Figure 2 shows a detailed diagram of the differentiating and amplifying circuits, and the monostable oscillator. The values of the components of the RC circuit are taken so that its output signal is approximately equal to the derivative of the input. Since the duration of the breakdown pulses during anodization at a given constant current is always below several tenths of a second, the values $C=1 \mu F$ and $R=470 \mathrm{kohm}$ were chosen. The amplifying circuit consists of an internally compensated 741 op. amp. with feedback through a $500 \mathrm{kohm}$ variable 


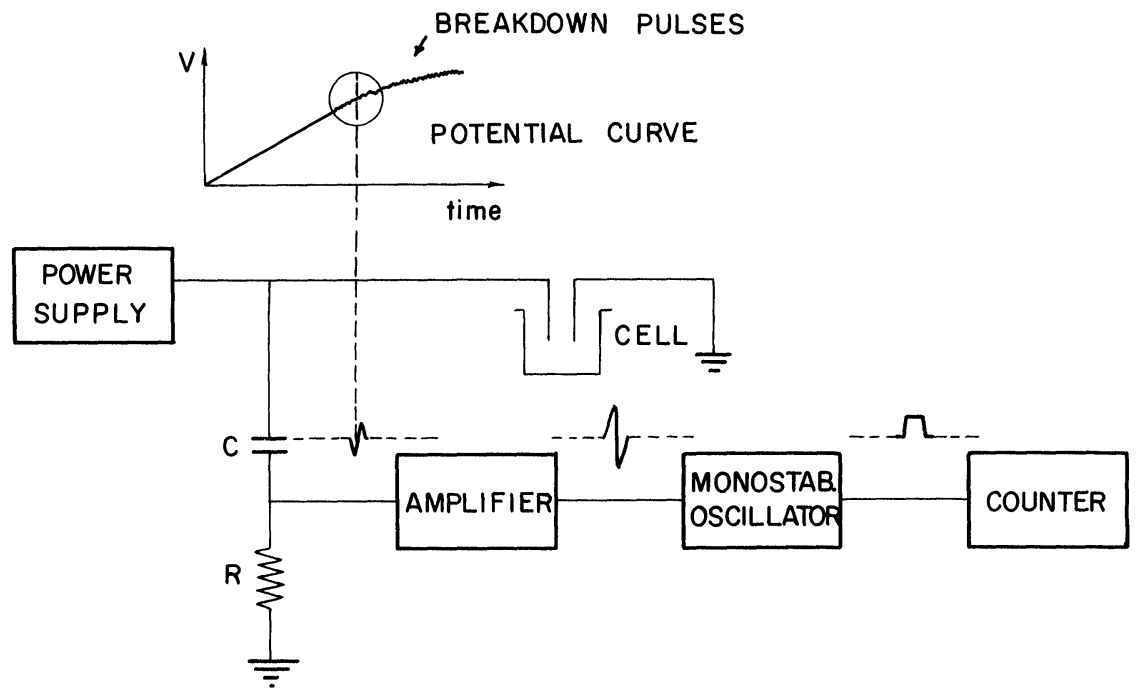

FIGURE 1 Block diagram of the electronic system for counting the breakdown pulses. The signal at each stage is also shown.

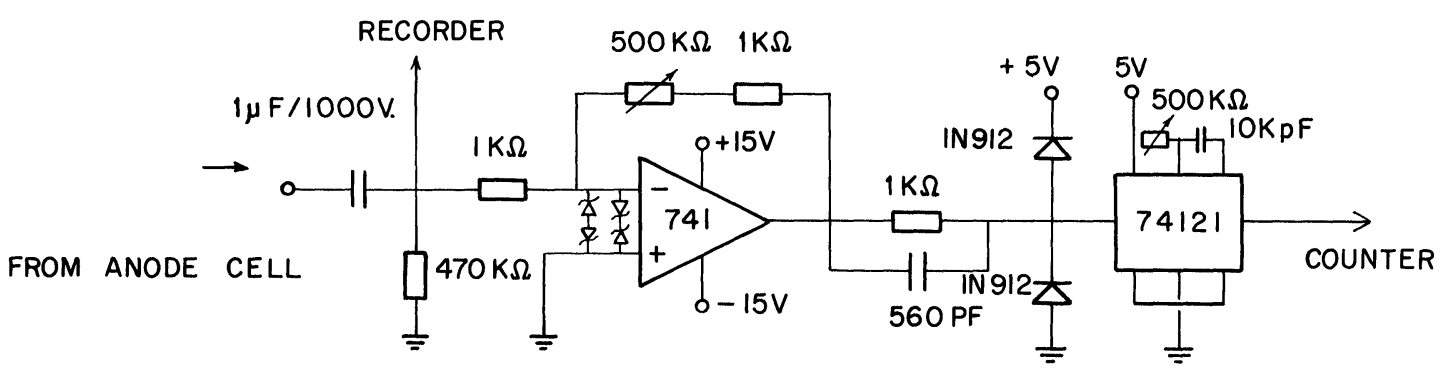

FIGURE 2 Schematic diagrams of the differentiating and amplifying circuits and the monostable oscillator.

resistor in order to vary the gain of the amplifier. A capacitor has been placed in parallel with the output resistor of the op. amp. so that its frequency response is flattened. The monostable oscillator is the 74121 non-retriggerable integrated circuit, producing a rectangular signal of variable width. The oscillator is able to discriminate between input pulses with a phase difference of $3 \times 10^{-6} \mathrm{~s}$. In the limit, the pulse counter, as a whole, is able to detect pulses as low as $0.1 \mathrm{~V}$ with a frequency of approximately $10 \mathrm{kHz}$.

\section{APPLICATION}

The apparatus described for determining the breakdown characteristics of anodic oxides has been successfully applied to etched aluminum foil, polished tantalum foil and pressed tantalum powder pellets, all of which constitute anodes currently employed in liquid or solid type electrolytic capacitors. As an application of the method, the results for tantalum foil will be described. The tantalum foil samples were first degreased with the standard solutions and subsequently chemically polished in a $5: 2: 2$ mixture of sulphuric, nitric and fluorhydric acids followed by an immersion in a $\mathrm{NH}_{4} \mathrm{~F}-\mathrm{HF}(300 \mathrm{~g} / \mathrm{l})$ solution. After the chemical polishing, the tantalum foil was thoroughly washed in boiling deionized water for 15 minutes. This method yields a very clean surface as can be checked by Auger electron spectroscopy. ${ }^{5}$ The breakdown experiments were performed in a $0.01 \% \mathrm{H}_{3} \mathrm{PO}_{4}$ electrolyte at $20^{\circ} \mathrm{C}$. A special device was designed in order to avoid sparking at the edges of the sample and at the electrolyte-sample-air interface which partially mask the real breakdown pulses. It consists of an insulating housing for the foil which allows only a 
portion of the surface of the foil (defined by means of a well pressed O-ring) to be in contact with the electrolyte, whereas the opposite surface of the foil, through which the electrical contact is made, does not touch the electrolyte. In this manner, the edge effects are avoided, the breakdown sparks appearing homogeneously across the sample's surface.

Figure 3 shows a typical example of the number of pulses registered by the counter as a function of the anodizing time for different current densities. It can be appreciated that the samples oxidized under a greater current density present a larger number of breakdown pulses. From the plot of Figure 4, it can be observed that the number of pulses per unit time, for a given anodization voltage, is also higher for the samples anodized with a greater current density. However, it is interesting to note that the first-spark voltage is approximately the same for all the samples, being independent of the current density. These results are in substantial agreement with those found by Kadari and Klein in sputtered tantalum films. ${ }^{3}$ Recently, we have developed a theory which qualitatively explains the above results. ${ }^{6}$ Basically, this theory assumes that, to the first approximation, all of the pulses consume the same amount of charge. This charge consumption appears as electronic, as opposed to ionic, leakage current. According to a formula given by Ikonopisov ${ }^{7}$ which is deduced from the breakdown theory of Forlani and Minnaja, ${ }^{8}$ the electronic current increases exponentially with the anodization voltage. Due to the fact that the

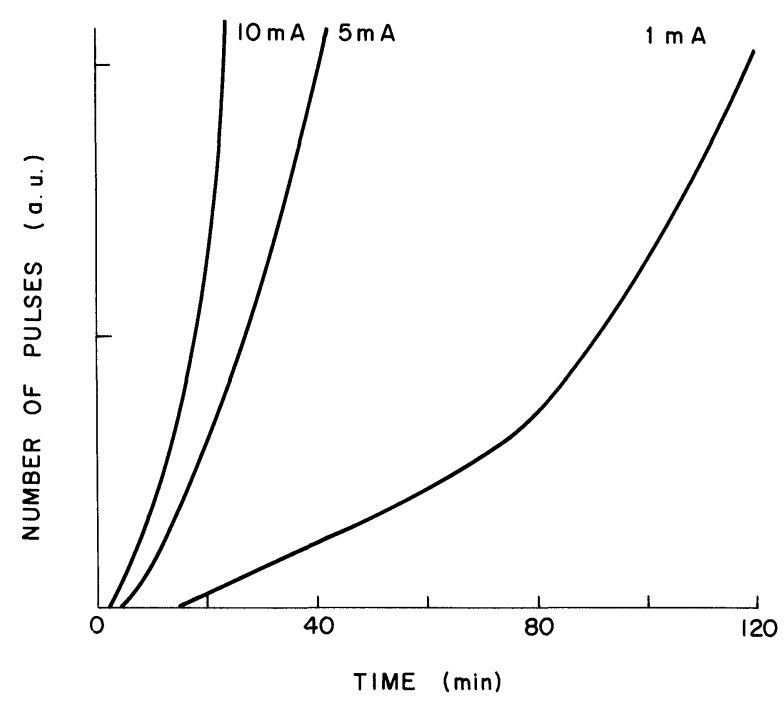

FIGURE 3 Number of breakdown pulses as a function of anodization time for a given constant current density.

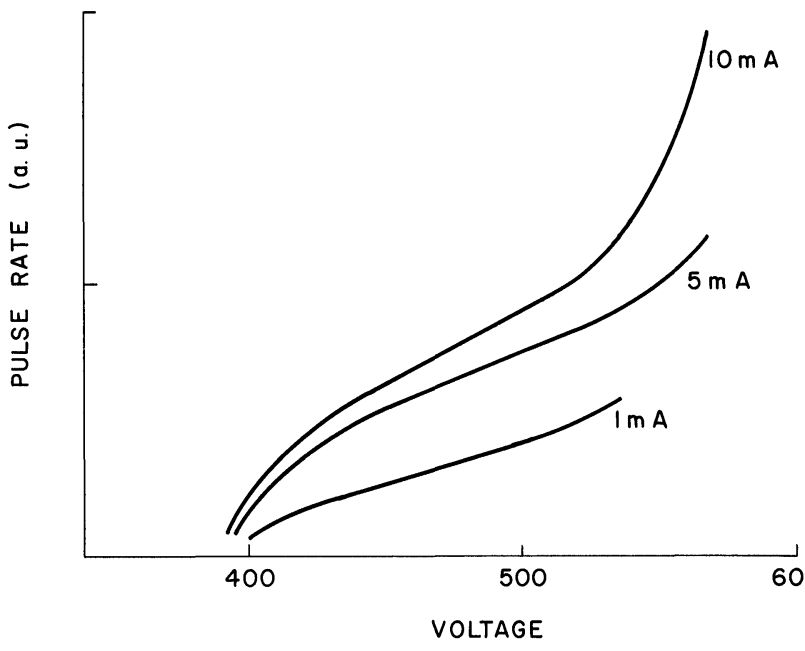

FIGURE 4 Number of pulses per unit time as a function of the anodization voltage for a given constant current density.

samples anodized with higher current density experience a faster voltage rise than those anodized with a lower current density, the number of pulses should increase faster with time in those samples oxidized with a higher current density, as observed in Figure 3. The proposed theory also predicts an exponential rise of the pulse rate with the anodization voltage, as shown in Figure 4.

It is not the object of this paper to make a detailed description of all the results that can be obtained with the pulse counter. It suffices to say that the apparatus is currently being used in our laboratory in order to determine the influence on the breakdown phenomena of different types of electrolytes, current density, temperature and resistivity of the electrolyte, etc. It is evident from the above results that the breakdown phenomenon strongly depends on the current density and that a measurement of the number of pulses during the anodization process can be very useful in elucidating the breakdown mechanisms.

\section{ACKNOWLEDGMENTS}

We would like to acknowledge PIHER, S.A. (Barcelona, Spain) and the American-Spanish Committee for Scientific and Technological Cooperation for partial support of this work, and Mr. J. Pedrosa for the mounting and checking of the electronic circuit. 


\section{REFERENCES}

1. F. J. Burger and J. C. Wu, "Dielectric Breakdown in Electrolytic Capacitors", J. Electrochem. Soc. 118, 2039 (1971).

2. J. Yahalom, "The role of the Film/Electrolyte Interface in Anodic Breakdown", in Oxyde-Electrolyte Interfaces, Ed. R. S. Alwitt, Electrochemical Soc. Inc. (Princeton) 289 (1973).

3. V. Kadary and N. Klein, "The Rate of Breakdown in Tantalum Pentoxide during Anodization" Proc. 8 th Israel IEEE Convention, (Tel-Aviv) vol III, 27 (1973).

4. U. M. Orlov, L. L. Odynets and T. I. Ryungenen, "Sparking during the Anodic Oxidation of Tantalum Containing Impurities" Elektrokhimiya 9, 818 (1973).
5. J. M. Albella, M. J. Puente, J. Baonza, M. T. Martin-Patino and J. M. Martinez-Duart, "Electrical Breakdown of Anodic Films on Tantalum", Proc. 7th Int. Vacuum Congress \& 3rd Int. Conf. on Solid Surfaces (Vienna), 2055 (1977).

6. J. M. Albella, I. Montero and J. M. Martinez-Duart, "Scintillation in Anodic $\mathrm{Ta}_{2} \mathrm{O}_{5}$ Films", Thin Solid Films 58, 307 (1979).

7. S. Ikonopisov, "Theory of Electrical Breakdown During Formation of Barrier Anodic Films", Electrochim. Acta 221077 (1977).

8. F. Forlani and N. Minnaja, "Electrical Breakdown in Thin Dielectric Films”, J. Vac. Sci. Tech. 6, 518 (1969). 

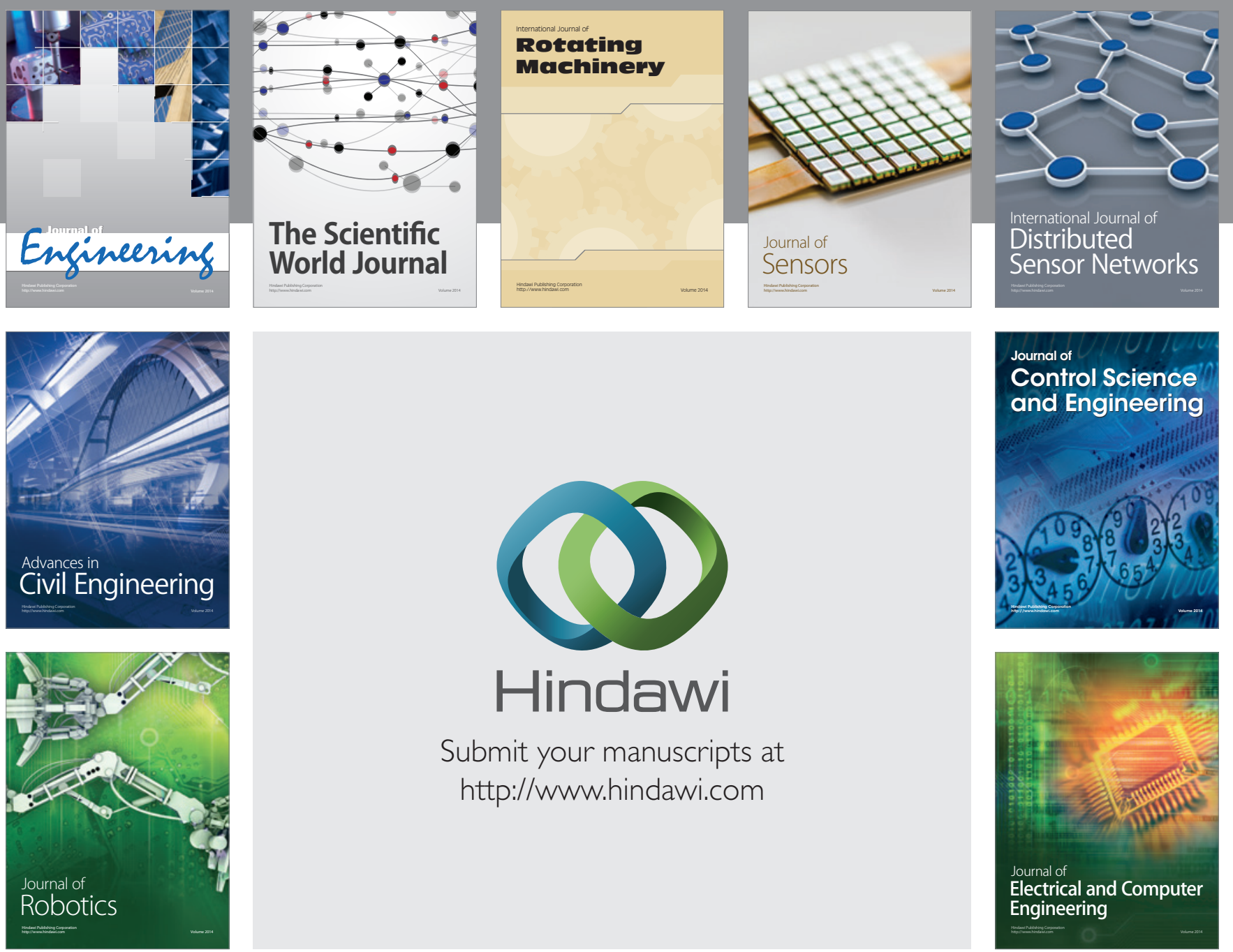

Submit your manuscripts at

http://www.hindawi.com
